\title{
Symmetry Fields of Palladian Villas
}

\author{
Matthew Swarts \\ Georgia Institute of Technology, USA \\ matthew.swarts@coa.gatech.edu
}

\begin{abstract}
In this paper we present the idea of symmetry fields and an analysis of them on Palladian villas. Symmetry fields describe the symmetry related to the localized experiential view of an individual. We propose that the amount of symmetry, whether it be reflective, rotational, or translational, can be computed along with several other measures of visual aesthetic. While many buildings are designed using symmetry about the plan, and are represented using a plan view, it is only accessible to the designer and cannot actually be experienced in the real world. The local symmetries produced are drastically different than those planned.
\end{abstract}

Keywords: Measureable aesthetics; Palladian villas; Space syntax; Neuroaesthetics; Computational aesthetics.

\section{Introduction}

While aesthetics is often thought of as the philosophy associated with judgments in art, in this paper, we focus particularly on the empirical aesthetic and neuroaesthetic perspectives that the sensation and perception of things can be observed and studied (Changeux, 1994; Fechner, 1871). In particular we focus on the symmetry aesthetic as one element in the formation of beauty. Symmetry, Greek for "to measure together", refers to a type of balance or of self-similar pattern, but also mathematically includes reflective, translational, rotational, and self-symmetry. The effects of bilateral symmetry are particularly strong in animals, as demonstrated in preferences for symmetric faces and bodies, which innately indicate a degree of healthiness and hence desirability (Møller \& Thornhill, 1998). Symmetries abound in nature and man-made patterns.

Symmetry has been used in the design of buildings since the beginning of architecture as one method to express the power of nature. In his Quattro Libri, Andrea Palladio, stated that the rules of architecture refer to the rules of nature. Palladio used harmonic proportions and rules of symmetry in his design of villas to express various aspects of beauty (Economou \& Swarts, 2006; Howard \& Longair, 1982). Through the use of these proportions, his plans typically express strong global symmetries. While the global symmetry is clearly defined, the impact of this global symmetry on the local spatial experience is unclear. Does the global symmetry propagate down into a local symmetry that can be experienced and understood by the user? In order to explore this further, we look to methods of computing the local experiential symmetry.

Several formulas have been developed to compute measures of aesthetics. Typically the computation involves some level of complexity divided by the ability to process that complexity (Birkhoff, 1933; Machado, Cardoso, \& de Oliveira, 1998). Others have focused on computing aesthetic measures specifically related to screen element layout and interface design (Altaboli \& Lin,
2011; Bauerly \& Liu, 2008; Ngo, Teo, \& Byrne, 2003; Pajusalu, 2012).

Our method for computing the experienced symmetry in an architectural context uses the concept of the isovist (Benedikt, 1979). The isovist is based on the sensation and perception of the information surfaces that surround an individual located in a particular space (Gibson, 1966). The main concept behind the isovist is that we can only experience the space that surrounds us at a given point in space. As we move through space we develop a cognitive map, but to do so, we use what we can see from one point to create an isovist. By computing the isovist in an array of locations on a plan, we can generate an isovist field. This field is composed of multiple isovists sampled at regular intervals within the given plan.

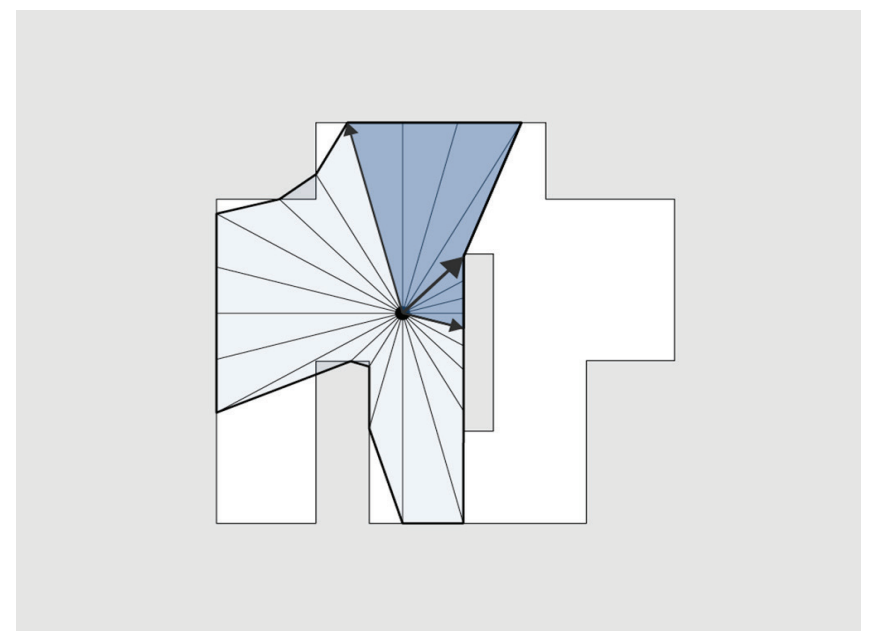

Figure 1: Illustration of a sampled isovist using 24 rays. The darker portion represents a partial isovist oriented in the direction of the larger arrow. Higher resolution reduces the approximation error. 
The isovist can be computed in several ways, but with high enough resolution, the differences between the true isovist and a sampled isovist (Figure 1) become negligible. In the case of Depthmap, a tool commonly used for space syntax analysis (Turner, Doxa, O'Sullivan, \& Penn, 2001), the similar idea as the isovist is produced through topological graph analysis rather than through ray tracing. The primary use in Depthmap is to identify which tiles can be reached in from the current tile. Consequently this is also an approximate measure of the amount of visible area from one point in space. The specific shape of the isovist is ignored, as only the area and the topology are of importance. For our work however, the specific shape of the isovist is the primary component of analysis.

\section{Methods}

We chose to use Palladian villas as our body of work from which to sample as proportion and global symmetry are known to be used in these plans. Our goal is to present a template for a computational method of aesthetic measures that can be used for architectural analysis. Specifically we modeled Villa Foscari, Villa Chiericati, and the Villa Rotunda in AutoCAD for analysis. Only the first floor of each building was used. The porticos were not included, and geometry was placed over the windows and exterior doors to create a fully enclosed set of indoor spaces. Additional lines were placed within the thick walls to create smaller areas which allowed for a simple method of excluding the insides of the walls from analysis. The plans were saved in a DXF format.

After importing each plan into our software, a grid of $30 \mathrm{~cm} \times 30$ $\mathrm{cm}$ tiles was placed over the plan. At the center of each tile, a sampled isovist was computed using 256 sample rays, giving an angular resolution of $\sim 1.4$ degrees. Tiles outside the plan, which included any isovist with a ray without an intersection, and isovists which had an area below a fixed threshold were excluded from further processing. The remaining isovists formed an isovist field for the plan.

The lengths of the rays of each sampled isovist was used to compute a reflection symmetry score for each tile. The reflection symmetry score was computed by first calculating the reflection symmetry difference error by summing the iterative (Figure 2) reflection difference error for each sample angle. The reflection difference error was computed by iteratively calculating and summing the square of the difference between corresponding ray sample lengths through a reflection about the sample angle. After all of the reflection symmetry difference errors were calculated for each tile in the isovist field, the values were normalized with respect to one another, and then subtracted from 1 . The plot of this score per sample angle can be visualized in the bottom histogram of Figure 2. Larger values correspond to more reflection symmetry at the sample angle. An overall symmetry score was calculated by summing the amount of matching symmetry at finer resolutions of detail. These scores were then visualized to produce an image of the symmetry field for a specific type of symmetry. The symmetry can be specified by point of regard and field of view, or overall score to allow for the comparison of different layouts. The tile colors were used for visualization of various normalized measures.

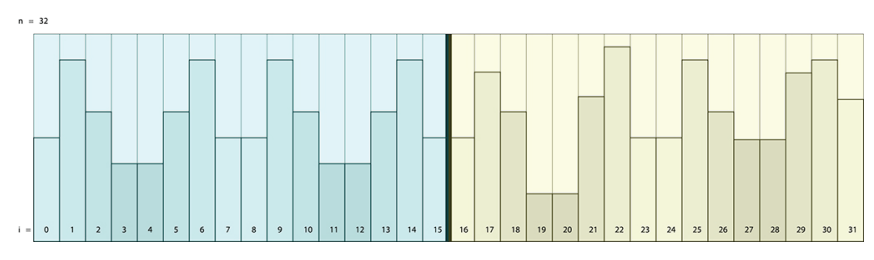

Figure 2: An illustration of how the values from histogram of a sampled isovist are used to pair with one another in computing the difference between two ray distance values about a particular sampled angle.
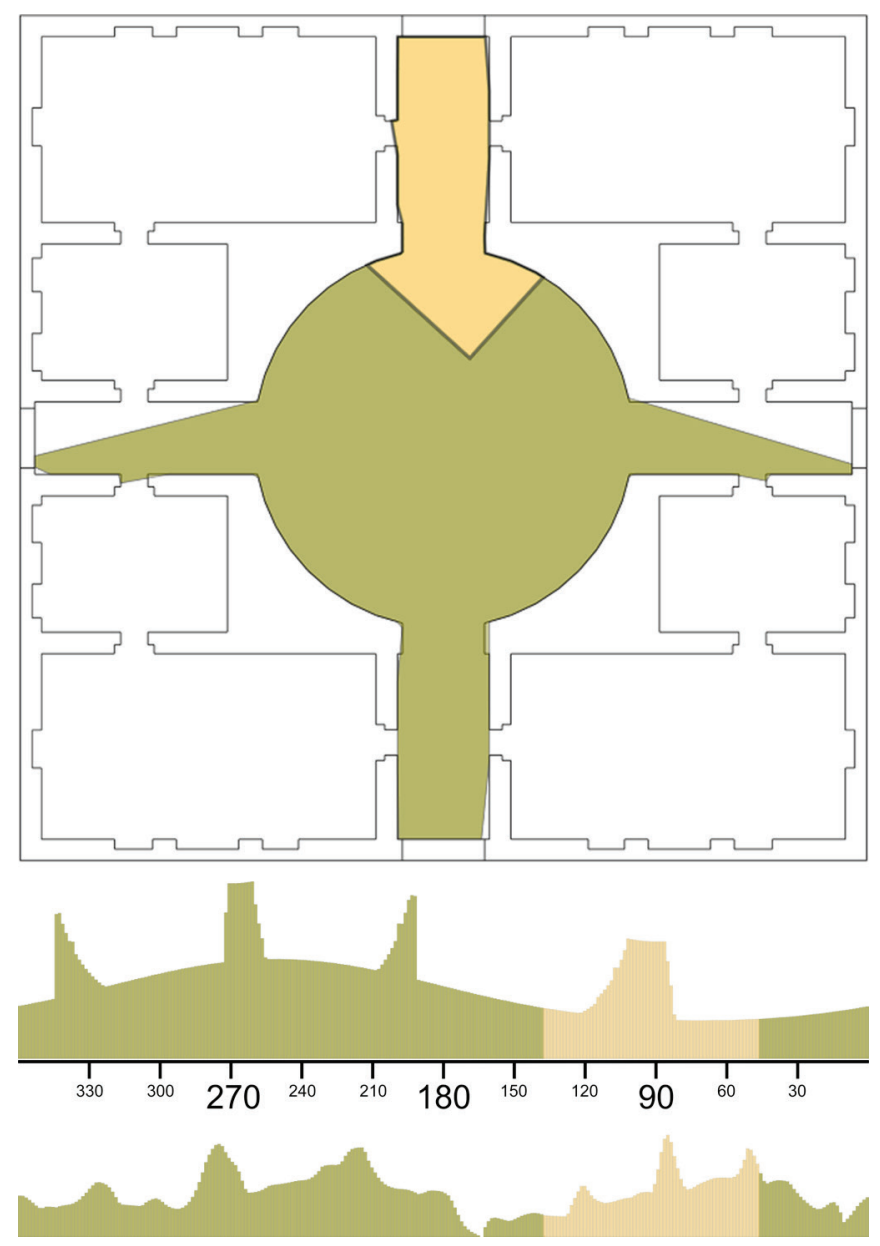

Figure 3: A sampled isovist in the plan of the Villa Rotunda. The lighter yellow portion is a partial isovist. The first graph below the plan is a histogram of the normalized ray traced distances associated with the sampled isovist. The lighter yellow portion maps to the partial isovist coverage. The bottom graph is a normalized reflection symmetry score at each sampled angle of the isovist. 

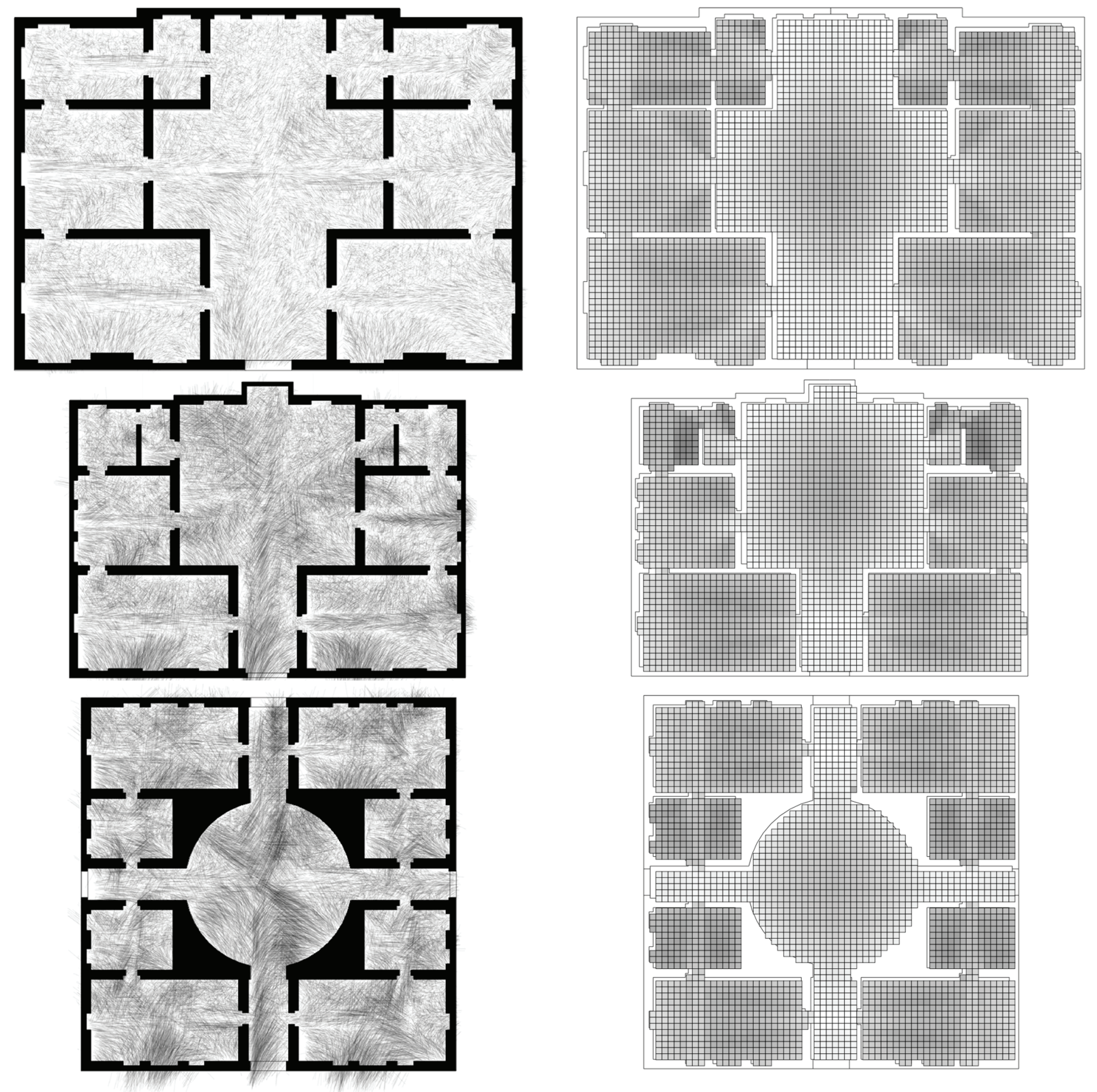

Figure 4: An illustration of the reflection symmetry 'forces' for each of the three villa plans (from top to bottom, Foscari, Chiericati, Rotunda). The forces are based on a probabilistic vector field across each plan.

While a single symmetry score can be produced for each isovist, it was also useful to analyze the symmetry scores for each sample angle. A typical vector field is created through a single vector direction and magnitude for each location along the grid of the vectors. Here the symmetry scores cannot easily be reduced to a single vector from each of the scores at the sample angles. Often there are several directions which are equally strong in magnitude of symmetry. Taking the maximum likelihood estimate would obscure much of the score. Instead we took the scores for each isovist as a vector probability distribution, using each score to

Figure 5: Tile based gradient visualization of the reflection symmetry scores, normalized by each of the three villa plans (from top to bottom, Foscari, Chiericati, Rotunda). The directions of the symmetry are not exposed in this visualization method.

build a frequency distribution. To visualize the vector probability distributions of the symmetry scores for each sample angle per isovist in each plan, we developed a modification of a line integral convolution method. Our method uses the values for each angle in a scored isovist to be used in producing a frequency distribution around the tile center. Fifty thousand lines are calculated by randomly placing points around each tile center, and then, using the vector frequency distribution, sampling for a vector direction and magnitude for the corresponding point (Figure 5). 


\section{Results}

The count, mean, and variance for the isovist areas and the isovist symmetry scores were calculated. The symmetry scores were normalized across all tiles by the maximum score in order to produce a gradient field through the tile colorization (Figure 4). Table 1 shows some of the available descriptive statistics across the three sample plans.

Table 1: Reflective symmetry score descriptive statistics for the three sample Palladian villas.

\begin{tabular}{|c|c|c|c|}
\hline & Foscari & Chiericati & Rotunda \\
\hline Tile Count & $4.178 \times 10^{4}$ & $2.558 \times 10^{4}$ & $2.946 \times 10^{4}$ \\
\hline Score Sum & $2.765 \times 10^{9}$ & $1.283 \times 10^{9}$ & $1.306 \times 10^{9}$ \\
\hline Score Mean & $6.619 \times 10^{5}$ & $5.018 \times 10^{5}$ & $4.432 \times 10^{5}$ \\
\hline Score Variance & $1.082 \times 10^{14}$ & $5.149 \times 10^{13}$ & $5.339 \times 10^{13}$ \\
\hline Area Sum & $6.116 \times 10^{8}$ & $2.335 \times 10^{8}$ & $1.980 \times 10^{8}$ \\
\hline Area Mean & $1.464 \times 10^{5}$ & $9.128 \times 10^{4}$ & $6.721 \times 10^{4}$ \\
\hline Area Variance & $1.908 \times 10^{13}$ & $4.786 \times 10^{12}$ & $3.000 \times 10^{12}$ \\
\hline
\end{tabular}

The higher score mean for Villa Foscari indicates that on average it contains slightly more experienced reflective symmetry, as per our definition, as compared to Villa Chiericati or Villa Rotunda. However, these gross values do not reveal as much as the force line visualizations, in which the symmetry scores directions are taken into account.

\section{Discussion}

In this paper we have introduced the concept of a symmetry score. We have shown a way to compute the aesthetic measure of localized experiential symmetry from a 2D plan. We have demonstrated our technique using three similar Palladian villa plans to illustrate the variation in local symmetry forces. This technique can be used to empower the designer with the ability to visualize the impact of an occupant's experience through local symmetries within a given design. By allowing these measures of aesthetics, we hope that we can begin to expand our ability to use computation for aesthetic evaluation.

In future work we will expand this notion to three dimensions using the Z-buffer commonly computed by a graphics card for visualization of 3D virtual environments. Additionally, this concept can be extended to additional computational aesthetic measures.

\section{References}

Altaboli, A. \& Lin, Y. (2011). Objective and Subjective Measures of Visual Aesthetics of Website Interface Design: The Two Sides of the Coin. In J. Jacko (Ed.), Human-Computer Interaction. Design and Development Approaches (Vol. 6761, pp. 35-44): Springer Berlin Heidelberg.

Bauerly, M \& Liu, Y. (2008). Effects of symmetry and number of compositional elements on interface and design aesthetics. International Journal of Human-Computer Interaction, 24(3), 275287. doi: $10.1080 / 10447310801920508$

Benedikt, M. L. (1979). To take hold of space: isovists and isovist fields. Environment and Planning B, 6(1), 47-65.

Birkhoff, G.D. (1933). Aesthetic measure: Harvard University Press.

Changeux, J.P. (1994). Raison et plaisir: O. Jacob.

Economou, A. \& Swarts, M. (2006). Performing Palladio. International Journal of Architectural Computing, 4(3), 47-61.

Fechner, GT. (1871). Zur experimentellen Aesthetik [Toward an experimental aesthetic]. Leipzig: S. Hirzel.

Gibson, J.J. (1966). The Senses Considered as Perceptual Systems.

Howard, D \& Longair, M. (1982). Harmonic Proportion and Palladio's "Quattro Libri". Journal of the Society of Architectural Historians, 41(2), 116-143. doi: 10.2307/989675

Machado, P., Cardoso, A., \& de Oliveira, F. M. (1998, 1998/01/01/). Computing aesthetics. Paper presented at the Advances in Artificial Intelligence. 14th Brazilian Symposium on Artificial Intelligence, SBIA'98. Proceedings, Place of Publication: Berlin, Germany; Porto Alegre, Brazil. Country of Publication: Germany.

Møller, A. P, \& Thornhill, R. (1998). Bilateral Symmetry and Sexual Selection: A Meta-Analysis. The American Naturalist(2), 174. doi: $10.1086 / 286110$

Ngo, C. L., Teo, L. S. \& Byrne, J. G. (2003). Modelling interface aesthetics. Information Sciences, 152(0), 25-46. doi: http://dx.doi.org/10.1016/S0020-0255(02)00404-8

Pajusalu, M. (2012). The Evaluation of User Interface Aesthetics. (Masters), Tallinn University.

Turner, A., Doxa, M., O'Sullivan, D., \& Penn, A. (2001). From isovists to visibility graphs: a methodology for the analysis of architectural space. Environment and Planning B: Planning and Design, 28(1), 103121. 